\title{
Genitopalatocardiac syndrome
}

INSERM

\section{Source}

INSERM. (1999). Orphanet: an online rare disease and orphan drug data base.

Genitopalatocardiac syndrome. ORPHA:2075

Genitopalatocardiac syndrome is a rare, multiple congenital anomalies/dysmorphic syndrome characterized by male, $46, \mathrm{XY}$ gonadal dysg enesis, cleft palate, microg nathia, conotruncal heart defects and unspecific skeletal, brain and kidney anomalies. 